Age-standardised/specific mortality and incidence rates (ASMR/ ASpMR and ASIR/ASpIR) of PLC subcategories were calculated. Trends in the rates of hepatocellular carcinoma (HCC) and intrahepatic bile duct tumours (IHBD) were evaluated using a regression method in which a least squares regression line was fitted to the natural logarithm of the rates. About $30 \%$ of incidence data for PLC included information on the ethnic origin of the cases and thus we were able to analyse the ethnic distribution of HCC and IHBD for this sub-set over the study period.

Results The ASMR for PLC increased in both sexes: from 3.88 and 2.03 per 100000 in 2001 to 5.10 and 2.63 per 100000 in 2008, for males and females respectively. Specifically, there was an increase in the ASMR for both HCC and IHBD of between $4 \%$ and $7 \%$ per year over the study period. ASIR of HCC increased in men (annual \% change: $4 \%$ ) but not in women. In the ethnic sub-set analysis, more than $79 \%$ of HCC and IHBD were registered in men and women of white ethnicity. Black Caribbeans and Africans, as well as Indians were the next most affected ethnic populations.

Conclusion Mortality and incidence rates of PLC continue to increase in England and Wales during 2001-2008, with a modest contribution from immigrant ethnic populations to the increasing trend.

Competing interests None declared.

\section{PWE-269 HEAT SHOCK PROTEIN-70 (HSP-70) A NOVEL SURROGATE MARKER FOR HYPOXIA INDUCED LIVER INJURY (HILI)-A PROSPECTIVE OPEN LEVEL CONTROL CLINICAL PILOT STUDY}

doi:10.1136/gutjnl-2012-302514d.269

\footnotetext{
${ }^{1,2} \mathrm{P}$ Basu, ${ }^{3} \mathrm{~T}$ Nair, ${ }^{3} \mathrm{~S}$ Farhat. ${ }^{1}$ Department of Gastroenterology and Hepatology, Columbia University of Physicians and Surgeons, New York, New York, USA; ${ }^{2}$ Department of Gastroenterology, North Shore University, New York, New York, USA; ${ }^{3}$ Internal Medicine, North Shore University, New York, New York, USA
}

Introduction Hypoxic hepatopathy $(\mathrm{HH})$ is a common entity in hospital with impaired hepatic perfusion secondary to transient altered cardio pulmonary haemodynamics, manifesting with sharp rise of transaminases with perfusion injury rarely requiring transplantation. Transaminases are non-specific index of reperfusion injury (IR). HSP are reperfusion signal proteins represent sheer stress. HSP-70 is one of specific signals of IR impacts on specific target. This study evaluates the utility of a novel marker of HILI. Methods Sixty $(n=60)$ patients were recruited from Hospital. Group A control $(n=20)$ Group B $(n=20)$ HILI, GroupC $(n=20)$ Acute Hepatitis [(Tylenol 8/20 (40\%), Acute hepatitis B 8/20 (40\%) herpes simplex $1 / 20$ (5\%) EBV 1/20 (5\%) acute hepatitis A 1/20 (5\%) unknown 1/20 (5\%)]. All groups underwent serial blood levels of HSP70, Liver and renal functions, MELD score, SOFA score, from day 0, 4, 7 and 10. Exclusion; Cardio respiratory failure, Renal failure, Acute alcoholic hepatitis, sepsis, organ transplant haemolytic Syndromes, CVA, MELD >20, MAP <90.

Results

\begin{tabular}{lllll}
\hline & $\begin{array}{l}\text { Group A } \\
\text { control } \\
\text { HSP level }\end{array}$ & $\begin{array}{l}\text { Group B hypoxic } \\
\text { liver injury } \\
\text { HSP level }\end{array}$ & $\begin{array}{l}\text { Group C acute } \\
\text { hepatitis } \\
\text { HSP level }\end{array}$ & $\begin{array}{l}\text { Group C } \\
\text { tylenol } \\
\text { HSP level }\end{array}$ \\
\hline Day & 0 & Intermediate & Low & Normal \\
Day 4 & 0 & Very high & Low & Intermediate \\
Day 7 & 0 & Very high & Low & Intermediate \\
Day 10 & 0 & Low & Low & Normal \\
MELD (mean) & 4 & 17 & 8 & 16 \\
SOFA (mean) & 2 & 8 & 4 & 6 \\
MAP (mean) & 122 & 98 & 102 & 124 \\
\hline
\end{tabular}

Conclusion Results: Group A; HSP 70 was normal. Group B was intermediate on day 0 and very high on day 4,7 and reverting back to lower levels on day 10. Group C had a low level (mildly elevated) of HSP-70 on all days with the exception of the Tylenol group where it was intermediate levels. Conclusion: HSP-70 is a relevant surrogate marker for hypoxia induced hepatopathy. HSP-70 levels strongly correlated with HILI and poorly with Tylenol induced liver injury. But negatively with other non-vascular liver injuries. Larger study needed to validate this finding.

Competing interests None declared.

\section{PWE-270 UNREVEALING A NOVEL ASSOCIATION OF CHOLESTEROL ESTER STORAGE DISEASE (CESD) AND NON-ALCOHOLIC FATTY LIVER DISEASE (NAFLD)—A SIMILAR CLINICAL SPECTRUM WITH DIFFERENT AETIOLOGY A PROSPECTIVE CLINICAL STUDY}

doi:10.1136/gutjnl-2012-302514d.270

${ }^{1,2} \mathrm{P}$ Basu, ${ }^{*}{ }^{3} \mathrm{~T}$ Nair, ${ }^{3} \mathrm{~S}$ Farhat, ${ }^{4} \mathrm{M}$ Jafri, ${ }^{3} \mathrm{~K}$ Mittimani, ${ }^{3} \mathrm{~N}$ James Shah, ${ }^{3} \mathrm{~L}$ Ang. ${ }^{1}$ Department of Gastroenterology and Hepatology, Columbia University of Physicians and Surgeons, New York, New York, USA; ${ }^{2}$ Department of Gastroenterology, North Shore University, New York, New York, USA; ${ }^{3}$ Internal Medicine, North Shore University, New York, New York, USA; ${ }^{4}$ Internal Medicine, NYMC Richmond, New York, New York, USA

Introduction NAFLD is the most evolving global morbidity progresses to cirrhosis, liver cancer and Transplantation. Clinical Spectrum is heterogeneous with biochemical and histological diversity. CESD is a part of metabolic storage disease with an intrinsic Lysosomal Acid Lipase deficiency (LAL) mimicking clinical overlap with NAFLD. This clinical pilot study evaluates the clinical overlap of similar metabolic syndromes with diverse aetiology And outcome.

Methods Three hundred $(\mathrm{n}=300)$ patients with fatty liver disease with Hepatomegaly, splenomegaly or Both with Mean BMI $27 \%$, Mean (Anthropometric assay W/H ratio mean 0.9, HDL 28, LDL 148, Triglyceride 187, HbA1c 5.9, HOMA Score 2.2, CRP 2.3, ALT 67, RBP 2.3, Homocystein 11, Leptin 3.6, Adiponectin 1.1 TNF- $\alpha$ 1,2, IL10 1.2, IL12 0.8. MELD 4). All under went Abdominal Sonogram and carotid artery Doppler. Serum Fibro sure, NASH score was measured and liver biopsy was performed in NASH group. Patients were divided into Group A $(\mathrm{n}=100)$ control with mean BMI $27.8 \%$ and no hepato-splenomegaly, Group B $(n=100)$ NAFLD with low BMI $<26 \%$, and Group C, NASH $(n=100)$ with NAFLD with BMI $>30 \%$.

Results

\begin{tabular}{lllr}
\hline & Group A & Group B & Group C \\
\hline LAL levels & 0 & $18 / 25(72 \%)$ & $7 / 25(28 \%)$ \\
Heterozygotes & 0 & $18 / 18(100 \%)$ & $6 / 7(86 \%)$ \\
Compound heterozygotes & 0 & 0 & $1 / 7(14 \%)$ \\
\hline
\end{tabular}

Conclusion Estimated prevalence of LAL $8.3 \%$ compared to the historical data (25 in 1 million) LAL has heterogeneity with an overlap WITH NAFLD and NASH. LAL deficiency has peripheral atherogenic potential with significant clinical morbidities with high Steatotic, Fibrogenic, and inflammatory scores than NAFLA or NASH. CESD is an integral part of Fatty liver disease.

Competing interests None declared. 